\title{
Prosthetic Rehabilitation of Nose After Partial Rhinectomy in a Patient Affected with Mucormycosis: A Case Report
}

\author{
Shrinidhi Jain ${ }^{1}$ (D) Satyabodh S. Guttal ${ }^{1}$
}

Received: 17 September 2021 / Accepted: 18 November 2021/Published online: 29 November 2021

(C) Association of Otolaryngologists of India 2021

\begin{abstract}
Mucormycosis is a fungal infection commonly seen in Indian population, in which aggressive surgical intervention to cut away the infected tissue becomes necessary. This may cause disfigurement and leave open wounds which requires prosthetic replacement for psychological benefit and also protect the inner soft tissues of the patient which are exposed to the foreign bodies. Mucormycosis has shown a sudden surge in recent times associated with patients affected by Covid-19. It is a rare but serious complication which can cause loss of eye, nose and associated structures leaving the patient disfigured. The aim of this case report is to describe an economic but effective nonsurgical treatment option to restore the facial defect using acrylic resin baseplate to enhance retention by using the available undercuts and was packed using silicone material. The prosthesis was retained mechanically and omitted the use of any retentive aids such as the use of spectacles or implants.
\end{abstract}

Keywords Mucormycosis - Nasal prosthesis .

Rhinectomy · COVID associated mucormycosis

\section{Introduction}

Mucormycosis is a rare fungal infection caused by mucoromycetes group of fungi affecting mostly the immunocompromised and rarely even the normal hosts [1]. The infection begins usually along the middle and inferior

Shrinidhi Jain

sjain7035@gmail.com

1 Department of Prosthodontics, SDM College of Dental Sciences and Hospital, Dharwad, Karnataka, India turbinate in susceptible individuals. Due to its angioinvasion, there is poor penetration of antifungal drugs to the affected site and its aggressive and acute nature necessitates aggressive surgical resection of the infected tissue. This disfigurement has pronounced psychological effect on the patient and severely affects the quality of life.

Coronavirus disease 2019 is a respiratory illness causing severe fever with associated symptoms. Individuals with known conditions such as diabetes mellitus or high blood pressure are more prone to developing mucormycosis [2] if they have been diagnosed with COVID-19 pneumonia. Around $80 \%$ of cases of Covid Associated Mucomycosis (CAM) were related to patients with pre-existing diabetes while $88.9 \%$ cases were mucormycosis of nose and sinuses and $56.7 \%$ of cases were rhino-orbital [3]. Many factors can increase the likelihood of mucormycosis. These include age, race, and previous trauma causing a break in skin.

Reconstruction of the defect can be completed via surgical reconstruction, prosthetic rehabilitation or a combination of the two, with retention of prostheses posing a major challenge [4]. Therefore a retentive prosthetic rehabilitation which is comfortable, esthetic and economic poses a great challenge to the prosthodontist. Here an acrylic baseplate was used [5, 6] below the silicone material as a base and provided sufficient retention to the prosthesis by engaging the minor undercuts. It also helps prevent complete blockage of nasal passages by preventing excess flow of silicone material while packing. 


\section{Clinical Report}

A 45 year old female patient was referred to the Department of Prosthodontics, SDM College of Dental Sciences and Hospital, Dharwad with a postoperative nasal defect caused due to surgical removal of affected structures with Mucormycosis. Patient resided in a village and had an agricultural background. Medical history revealed that she underwent a surgery 7 years back in the area of nose. The patient was a diabetic and came back again after 7 years with the symptom of pain in the same region and was diagnosed with mucormycosis after fungal culture. Surgical resection was performed to limit the further spread of mucormycosis infection. The septal cartilage, the left and the right lateral cartilages, right ala of the nose and part of the nasal septum were resected. The range of treatment options for replacement of the defect were explained to the patient. Due to time and economic constraints, a silicone nasal prosthesis was planned without the use of additional retentive elements like Implants. A written consent was obtained from the patient regarding the treatment plan and its outcome and prognosis.

The patient was made to sit on a dental chair which was reclined at a $45^{\circ}$ angle. Petroleum jelly was then applied on the eyebrows, eyelashes and surrounding areas. A suction tip, cut to 2 inch length was placed in the left nostril to prevent the displacement of the ala and flow of the material into the nostril of the patient during impression making procedure. The deep undercuts were blocked using moist gauze. The patient was encouraged to breathe through her mouth and impression was made using a light body polyvinyl silicone material (Aquasil LV Ultra light body cartridge, Dentsply sirona) which was used to accurately record the defect site and also cover normal tissues (Fig. 1). Then the material was reinforced using Putty consistency Polyvinyl silicone material (Aquasil soft putty/ regular set, Dentsply). The impression was carefully removed and then poured with type-IV gypsum (Elite Rock thixotropic, Zhermack). Deep undercuts were then blocked on the retrieved cast using modelling wax and self cure clear acrylic (DPIRR cold cure, Mumbai) was mixed and adapted to the defect site. It was allowed to set, then retrieved and trimmed to a uniform thickness of $2 \mathrm{~mm}$. The acrylic baseplate was polished using a sandpaper of 120 grit size. The acrylic baseplate was placed on the cast and checked for the accuracy of fit and retention (Fig. 2). Once satisfactory, wax was moulded on the acrylic baseplate according to patient's descriptions of her preoperative appearance and the knowledge of normal anatomic contours [7]. The wax pattern was verified on the patient (Fig. 3) and flasked using Type-II plaster/ dental plaster. Dewaxing was done by immersing the flasks in boiling

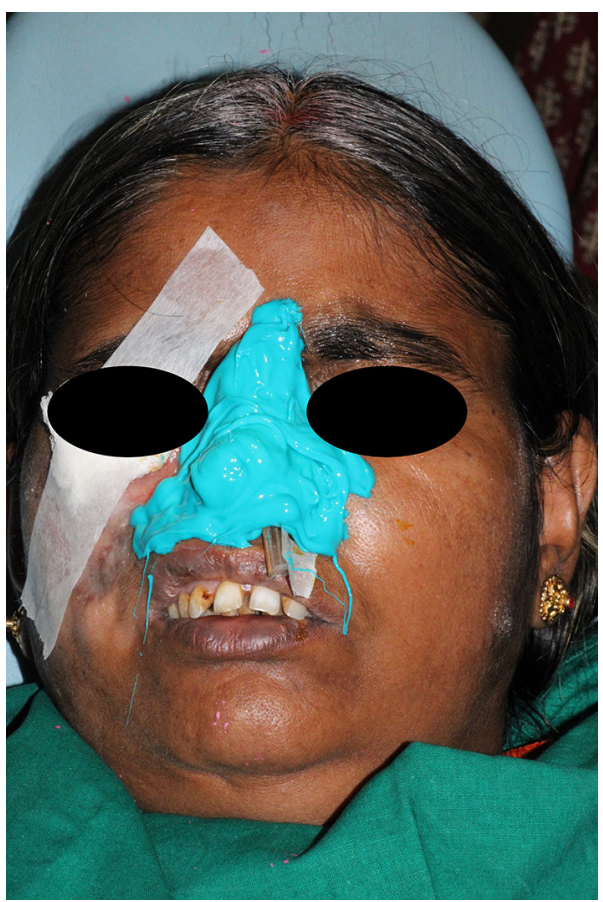

Fig. 1 Polyvinyl silicone (light body) impression keeping airway patent

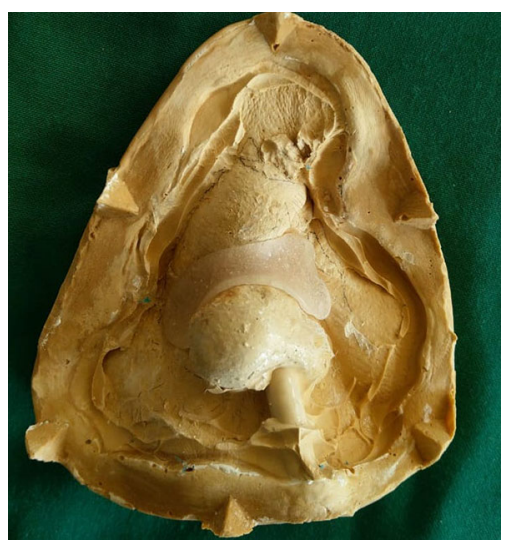

Fig. 2 Type II gypsum mould poured and fabrication of acrylic shim

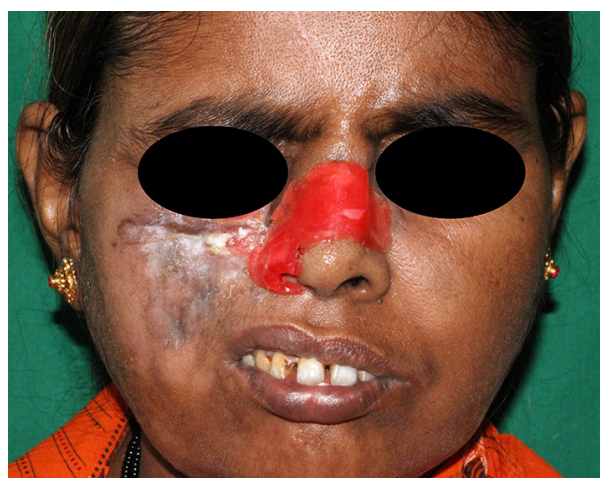

Fig. 3 Wax pattern fabrication 
water for 4 min (Fig. 4). The flasks were opened and wax was eliminated using hot water. A primer was then evenly applied on the acrylic baseplate so that silicone could bind to the acrylic material (Universal tray adhesive, Zhermack). Room Temperature Vulcanising Silicone (Silicone for prosthesis, MP Sai enterprises, Thane) was mixed with intrinsic stains and colour matching was done. Different shades of silicone was packed according to the shade of the natural skin adjacent to the prosthesis. The flask was clamped and kept for $24 \mathrm{~h}$ so that the material sets sufficiently. It was retrieved the next day, the excess was trimmed and prosthesis was verified on the patient for retention (Fig. 5). Extrinsic staining was done to closely mimic the adjacent pigmentation on the skin. The prosthesis was delivered to the patient and home care instructions were given (Fig. 6).

\section{Discussion}

Mucormycosis also called previously as Zygormycosis is a rare but severe life threatening fungal infection commonly seen in Indian population. It is present in the environment, specially in the soil, manure, cowdung and though it is inhaled by most people, it only manifests in severely immunocompromised patients since phagocytes are the major host defence mechanism which can kill the fungi after it is inhaled through the nostrils [8]. The risk factors include uncontrolled diabetes mellitus, HIV, organ transplant patients receiving immunosuppressants, trauma, burns, malnutrition. Once there is fungal invasion into the blood vessels, it results in the formation of blood clots and surrounding tissue death due to a loss of blood supply [8]. Therefore systemic drugs do not work on the infected site as there is no blood supply to the affected site. Hence extensive surgery for removal of the fungus ball becomes necessary. Surgery involves removal of the adjacent normal tissues to prevent recurrence due to its aggressive nature of spread through the blood vessels.

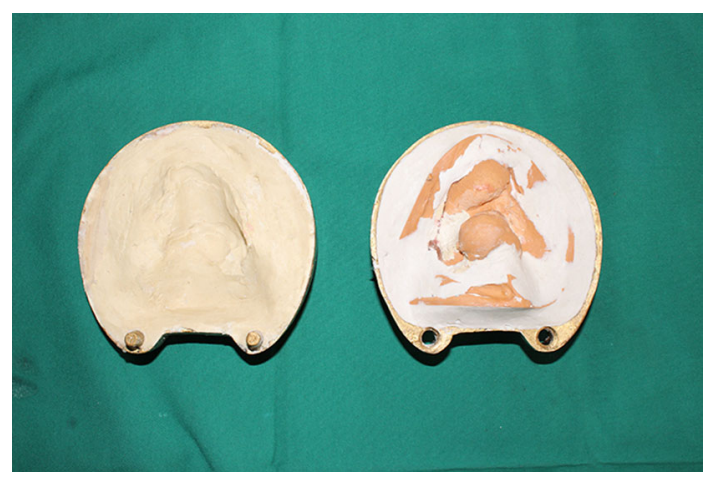

Fig. 4 Flasking of the pattern with two pour technique

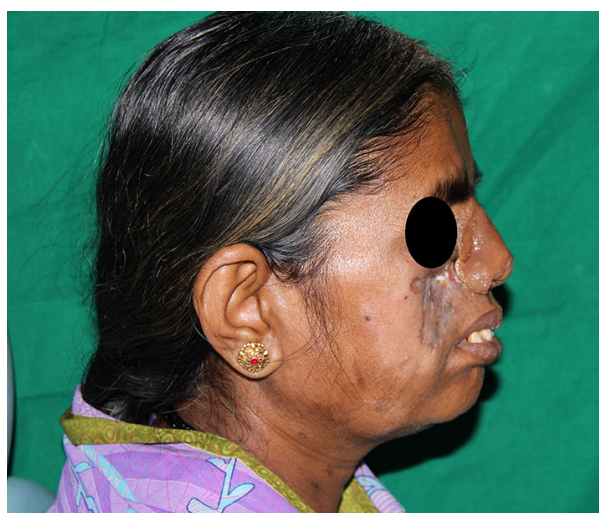

Fig. 5 Tryin of the fabricated silicone prosthesis

After rhinectomy, the quality of life is severely compromised if a prosthesis or surgical reconstruction is not provided. Since the nose is a prominent feature of the human face, nasal defects produce severe cosmetic impairment, when compared to other facial defects [9]. Construction of a good nasal prosthesis requires prosthodontic and artistic skills to provide adequate function and esthetics. The most important function of maxillofacial prosthesis is to provide protection to the remaining hard and soft tissues while also providing comfort to the patient [10]. Even though there are many other retentive means for a prosthesis such as implants, their use is restricted depending on the economic constraints and the medical background of the patient. It is advised therefore that before implant therapy, the prevalent systemic conditions should be controlled and there should be proper assessment of medical conditions [11]. Implant placement in such patients should be planned well because they are already immunocompromised. The monitoring becomes difficult in such patients after implant placement if they do not co-operate. For the purpose of prosthetic rehabilitation of facial defects, biomaterials such as polymethyl methacrylate, polyvinyl chloride, polyurethane and silicone have been used [12]. Silicones gained more popularity through the years due to its good physical properties, excellent biocompatibility and good thermal stability. They can be extrinsically and intrinsically stained and is sanitary.

\section{Summary}

As there is a rise in the number of patients affected by Mucormycosis every year [13], there might be increased demand for post operative prosthesis fabrication. Nasal prosthesis along with providing psychological benefit, also has a protective function by acting as a barrier to foreign particles which may directly come in contact with the nasal 
Fig. 6 Insertion after extrinsic staining

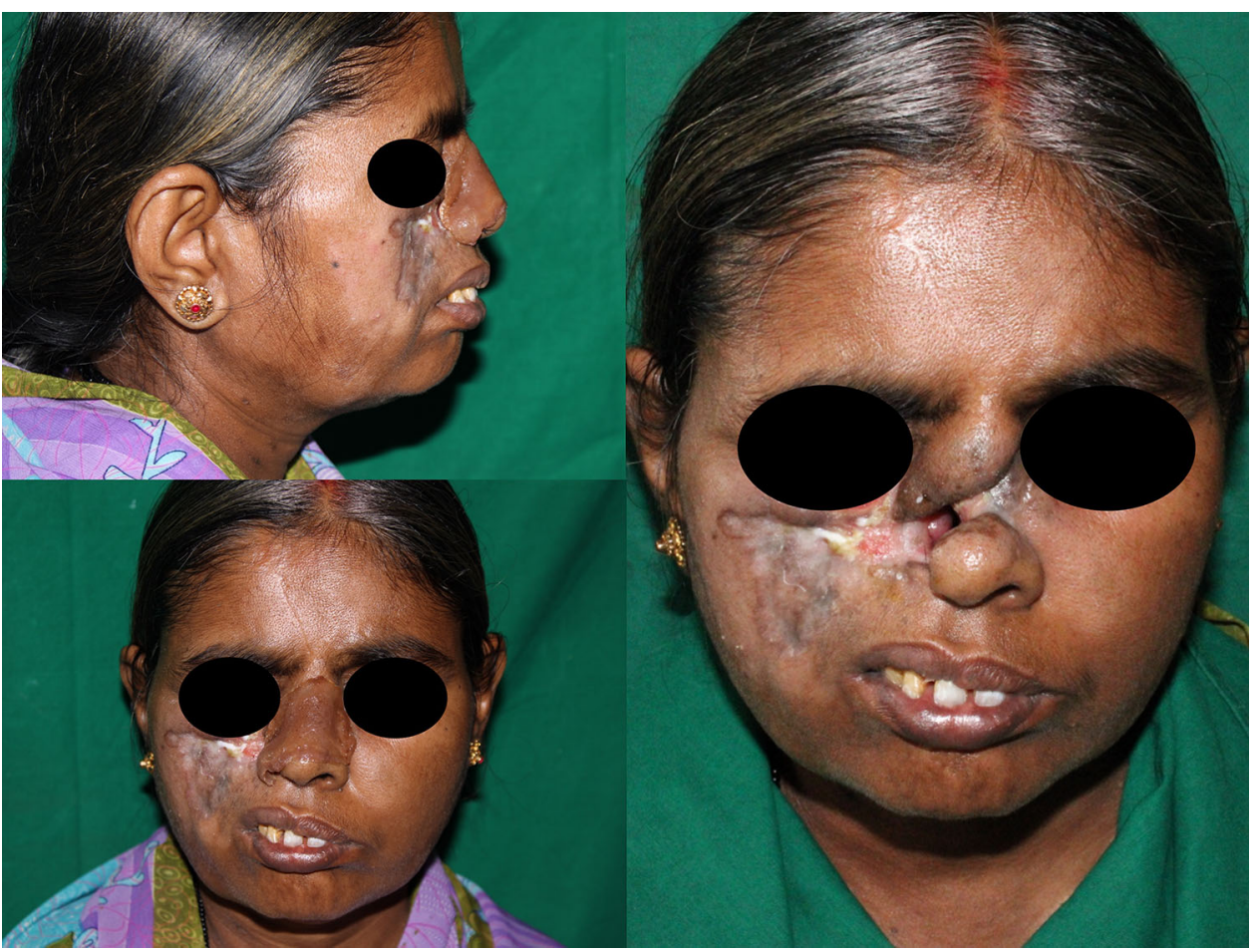

mucosa and further cause complications in immunocompromised patients. Although many other prosthetic treatment options might be available, such a treatment is most opted by patients as it is economical and is not time consuming.

\section{References}

1. Ferguson BJ (2000) Mucormycosis of the nose and paranasal sinuses. Otolaryngol Clin N Am 33(2):349-365

2. Hagen A (2021) COVID-19-associated mucomycosis: triple threat of the pandemic. American Society for Microbiology. https://asm.org/Articles/2021/July/COVID-19-AssociatedMucormycosis-Triple-Threat-of. Accessed 15 July 2021

3. Singh AK, Singh R, Joshi SR, Misra A (2021) Mucormycosis in COVID-19: a systematic review of cases reported worldwide and in India [published online ahead of print, 2021 May 21]. Diabetes Metab Syndr. https://doi.org/10.1016/j.dsx.2021.05.019

4. Brunski JB, Puleo DA, Nanci A (2000) Biomaterials and biomechanics of oral and maxillofacial implants: current status and future developments. Int $\mathrm{J}$ Oral Maxillofac Implants 15(1):15-46

5. Walma D, Seidenfaden J, Mourad F, Kase M (2019) Prosthetic rehabilitation via silicone nasal prosthesis with internal alar support following partial rhinectomy: a short communication. Int J Prosthodont 32(2):208-210

6. Muttlib NA, Fadzlina AK (2018) Construction of silicone nasal prosthesis with acrylic baseplate. Otorinolaringologia 68(3): 108-110

7. Brown KE (1971) Fabrication of a nose prosthesis. J Prosthet Dent 26:543-554

8. Spellberg B, Edwards J, Ibrahim A (2005) Novel perspectives on mucormycosis: pathophysiology, presentation, and management. Clin Microbiol Rev 18(3):556-569

9. Breitbart W, Holland J (1988) Psychosocial aspects of head and neck cancer. Semin Oncol 15:61-69

10. Dhiman R, Arora V, Kotwal N (2007) Rehabilitation of a Rhinocerebral mucormycosis patient. J Indian Prosthodont Soc 7:88-91

11. Akay AS, Arısan V (2018) Dental implants in the medically compromised patient population. In: Clinical trials in vulnerable populations. London

12. Reports of Councils and Bureaus (1975) Maxillofacial prosthetic materials. J Am Dent Assoc 90(4):844-848

13. Chakrabarti A, Dhaliwal M (2013) Epidemiology of mucormycosis in India. Curr Fungal Infect Rep 7:287-292

Publisher's Note Springer Nature remains neutral with regard to jurisdictional claims in published maps and institutional affiliations. 